International Journal of Electrical Engineering and Technology (IJEET)

Volume 11, Issue 4, June 2020, pp. 257-265, Article ID: IJEET_11_04_029

Available online at https://iaeme.com/Home/issue/IJEET? Volume $=11 \&$ Issue $=4$

ISSN Print: 0976-6545 and ISSN Online: 0976-6553

https://doi.org/10.34218/IJEET.11.4.2020.029

\title{
STAKE HEALTH MONITORING SYSTEM EPITHETICAL ON IOT AND CLOUD AN EFFICIENT DATA STOCKPILE
}

\author{
Geetha K, Chaithanya B N, Dr. Dayanand Lal N and Neetha K S \\ GITAM - Gandhi Institute of Technology and Management (Deemed to be University), \\ Department of Computer Science Engineering, Bangalore, Karnataka India
}

\begin{abstract}
IoT is a heterogeneous network of smart connected devices - each of which collect and share data about how they are used and the environments in which they are operated. The IoT network consists of sensors / devices transmitting data to the cloud through various device-based communication technologies. Post uploading the data to the cloud, software processes the data and can then start taking an action such as sending a notification or updating the sensors / devices automatically without a manual intervention. Innovation assumes that the important and significant element in medicinal activities or services for substantial or substantial contraptions as well as in affiliation with affiliation, recording and appear contraption. It is crucial to show or screen distinctive therapeutic parameters conjointly post operational days. Here after the foremost advanced or later design in Healthcare specialized too master strategy utilizing IOT is balanced the microcontroller gets the sensor data and sends it to the framework all the way through Wi-Fi and subsequently gives persistent watching of the human administrations or exercises parameters for master or masters. The data can be gotten to whenever by the pro. The controller to boot related with flag to caution the supervisor almost assortment in sensor surrender. However, the noteworthy issue in farther understanding checking system is that the data has to be securely transmitted to the objective conclusion and course of action is made to empower fair affirmed client to induce to the information.
\end{abstract}

Key words: Remote Health Monitoring, Wearable Sensor, IOT, Medical Analyst, Framework.

Cite this Article: Geetha K, Chaithanya B N, Dr. Dayanand Lal N and Neetha K S, Stake Health Monitoring System Epithetical on IoT and Cloud an Efficient Data Stockpile. International Journal of Electrical Engineering and Technology, 11(4), 2020, pp. 257-265.

https://iaeme.com/Home/issue/IJEET?Volume=11\&Issue $=4$ 


\section{INTRODUCTION}

Web has changed people's lives drastically. It has become a huge part of our lives and changed a lot of things, from how we live, learn, and work. As of today, IOT systems rely mainly on sensors and remote systems to allow access to data or applications wherever and whenever possible. In any case, IOT provides more than any other sector significant assurance in the area of wellbeing consciousness. As a maximum goes "Wellbeing is riches" it is astonishingly significant to create Utilize of the advancement for superior success. The provisioning and avail oneself of these IOT devices opens door to new business opportunities. Industry and academia must handle these networked devices and take advantage of the enormous amount of data generated. Hence the IOT system offers a secure and comprehensive regulation of prosperity for Wellness is affluent.

The monitor screen systems in mending offices constantly search for persistent simple signals that allow the sensors to be hardwired to be available, the displays or computers close the bed of the patient and restrict comprehension of the station that is allocated by the specialist. Without a doubt, indeed consequent to partner these systems to a particular understanding, a paramedical accomplice ought to always screen and note down all the key parameters of a given persistent by observing the larger part of his/her records physically. Grasping such a method is botch slanted and may incite disaster on account of a human bumble.

Data sensing is carried out by specific wearable sensors for instance, ECG, EMG muscle activity, skin temperature and respiratory rate graders physiologic biomarkers. In order to monitor the health of the patient, they will be implanted with various wearable devices which will have sophisticated sensors like example ECG sensor, Body position sensor, Airflow sensor, Body temperature sensor, EMG sensor, Glucose sensor, Galvanic skin sensor, Blood pressure sensor that are capable of collecting the diverse patient data which indicates the current health condition of the patient. All these wearable devices are not only capable of collecting data but also designed to transmit data to the concentrator via communication medium such as Wi-Fi, ZigBee, Bluetooth, Zwave etc. This collected huge data will be stored in the cloud, since cloud provides efficient framework for storing and accessing the data remotely. Health analytics gather and analyze data in the field of healthcare to get perspective and to promote decision making. Health analytics at simple and complex level can be used efficiently to standardize processes, strengthen patient care and reduce overall costs in varying key areas such pharmaceutical, patient condition monitoring, health details, medical expenses. The health analytics is intended to amalgamate all information from the electronic health records which provides exhaustive diagnosis and treatment for more persuasive care. Analytics need to predict uncertainty when taking into account a patient's numerous medical conditions. The healthcare industry will deploy resources more efficiently to actively engage with high risk patients early on by consolidating and reviewing all these types of evidence and avoiding systematic loses for long term.

Long afterwards, they saw a spike eagerness for wearable sensors devices and nowadays a number of contraptions are monetarily available in some research papers for person human administrations, fitness, and activity mindfulness. Considering the current rate of advancement in mechanical designs, we can expect the physiological checking utilizing conservative wearable sensors to cease entirely sooner rather than later. The advancement will increase the precision of analysis of therapeutic administrations systems and also makes them faster.

\section{LITERATURE SURVEY}


Alexandros Pantelopoulos et.al. [1] has expressed a concept on design and development of wearable bio-sensor structures for health monitoring has gathered information paying attention in the scientific communal as well as in industry from few decades there has been lots of changes in IoT-based social comfort enhancement presentations in the past decade. As the cost of healthcare is getting increased and the world population is dragging more, there is a necessity to keep track an individual patient's healthiness position if at all patient is isolated or quarantined environment. To indicate this purpose, various types of system prototypes and commercial goods have been produced in the sequence of fresh years, which target at in case real-time feedback data about individual health illness, either to the consumer himself or to a medical center or straight to a administering professional physician, while being able to alert the single in case of conceivable imminent health frightening conditions.

Aleksandar Kotevski et.al. [2] articles has spoken about the Modern information and communication technology, granting access everywhere and anywhere, play a vital role in the advancement of modern healthcare systems. Nowadays, there are numerous electronic platforms for tracking and gathering patient data. Such solutions are especially useful when a treatment involves monitoring some vital parameters for a long time.

The e-health structure, presented by the writers, will be used to track key metabolic data of patients. It consists of three major components: Web, phone and Smart TV subsystem, trying to cover the key features of a distant healthcare monitoring system. The device would gather essential data required and make it visible to doctors.

Jin et al. [3] projected a framework structure for making IoT smart city executions, as well as brilliant human services. As per this article, there are three fundamental perspectives that guide the working of an IoT execution: organize driven IoT, cloud-driven IoT and information driven IoT. Each view point has a few building squares, and each building deter in a point of view relates to at slightest one pieces in substitute viewpoints. The three viewpoints strongly associated with each other significantly impacted the operation of IoT in the very same way. Following developments in computer social issues, management and remedial data review and oversight remain central components of unavailable prosperity control schemes.

The right decisions and reviews on the patient's medical condition rely on the restorative documentation with various clinical characteristics being examined over a period of time. IoT devices could be unsafe, complicated and improper to use. They can only be used to do conventional tasks like identifying goals and exchange information with each other. Also exchanging information with prosperity foundations using internet will improve the organization assignments.

According to M. Fenrou G et.al [4] have developed a design on health monitoring system which works in lower bandwidth for rural area, but large volumes of the generated data need to be stored and analysed efficiently. Studying the network communication requirements of a remote health monitoring application that is based on IOT. Proposing a Centralized overall safety control system for analysing and comparing different network communication protocols. Converting the architecture into a prototype that can reduce the volume of data generated for a single sensor event by up to 90 per cent, and the bandwidth required for a healthcare scenario by up to 56 per cent compared to an existing commercial product.

D. Lake, $\mathrm{R}$ et.al. [5] have conversed few aspects of providing confidentiality, integrity and authentication for patient information deposited in cloud. Their projected system introduces security of healthiness care and cloud of things. System works in two major parts viz. storage stage and data retrieving stage. In storage stage, data is stored, updated for upcoming use. In information retrieving stage, recover data from cloud. The cloud server can segment with authenticated user as per request. 
Chehri, et.al. [6] have pondered different systems which can be utilized to assemble information from sensor organize. As remote innovation turns out to be progressively inescapable, e-wellbeing experts are thinking about remote systems for their portable medication frameworks with the approach of e-social insurance, a wide scope of advancements would now be able to be applied to give clinical consideration items and administrations. the creators have assessed shrewd sensor organize design for e-wellbeing applications. This design dependent on different corresponding remote interchanges get to systems between the patient and the framework, through UMTS, WiMax, and the Internet.

Sneha N. et.al. [7] has a healthcare system should give better healthcare services to people anywhere in an affordable and patient comfortably manner. Recently, the healthcare system is going to change from a old approach to a new patient centred approach. In the old way the doctors perform the main role. For necessary diagnosis and advising they need to visit the patients. The two basic problems related to this approach, very first the doctors compulsory should present at place of the patient for 24 hours and second, in the hospital, the patient remains admitted, wiring connected biomedical instruments to bedside, for long period of time. The patient friendly approach has received to solve these two problems. In this theme, the patients are aware with knowledge and information to play a more active role in disease diagnosis, and prevention. The important element of this second approach is a reliable and readily available patient monitoring system (PMS).

M. Sathya et.al. [8] expressing their views on IOT which plays a vital role in monitoring patient's health system. The compact sensors with IOT that are embedded in patient's body collect physiological data and transmit to the health center with accuracy and security. Using the available data records and decision support systems, the physician can make a much better prognosis and can suggest early treatment and we can see some previous works are address that there is a limitation of database connectivity between the different cloud environment in monitoring the data in constant time intervals and to analyze data. Considering this limitation, the authors has presented a cloud-based Internet of Things system that can be implemented in different health monitoring In the recent years wireless technology has increasing for the need of upholding various sectors. Modern health care system introduces new technologies like wearable devices or cloud of things. It provides flexibility in terms of recording patients monitored data and send it remotely via IOT.

\section{REMOTE HEALTH MONITORING SYSTEM BASED ON IOT}

\subsection{Architecture}

Fig1 illustrates the framework for the observation system of wellness, whose actual components we inevitably portray: In order to track health and continue to offer clinically appropriate care data, wearable sensors as their name implies, have been incorporated into wearable objects or directly with the patient body.

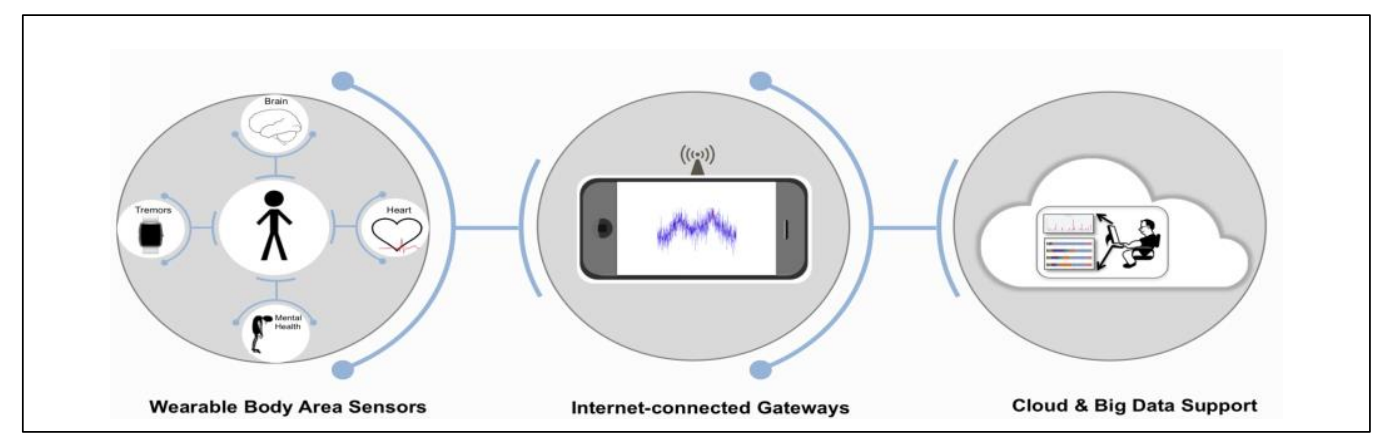


Figure 1 Remote health monitoring framework

The sensors interface with the framework be that as it may a center of the street data aggregator or concentrator, which is regularly a PDA arranged within the locale of the persistent. Contains domestic computerization contraptions, for illustration, sensors (temperature, dampness, smoke and CO, water gap) and actuators (control switch, jolt, and dimmer). In conclusion, IoT sensors, RFID sensors, accelerometers, can be joined to this layer with the purpose of further testing of prosperity. Today, the bio sensors or social security sensors are impressively rediscovering, including body temperature, circulatory strain, pulse, ECG, breathing rate, glucose level. A further source of information from human administration may start with cell phones that almost get coordinated as the entry of the farther local body. It is conceivable to accumulate all the wellbeing correlated data in this detecting layer and frontward it to higher level layers for additionally handling the region of versatile customer, here and there alluded to as a cloudlet, is utilized to expand its stockpiling/preparing capacity at whatever point the neighborhood portable assets don't satisfy the application's necessities . Cloud Processing has three particular segments: stockpiling, investigation, and perception. The framework is intended for long haul stockpiling of patient's biomedical data also helping wellbeing experts with demonstrative data. The cloudlet may be an adjacent area (for example, an individual computer) planning unit that is easily accessible via WiFi via a concentrator. Data are transmitted from the domestic entrance to the watch side, where the framework system is used. To date, unusual frameworks are open, specific cells $(3 \mathrm{G}, 4 \mathrm{G})$, convenient broadband, fiber and ADSL. WiFi and Ethernet propels can be combined by that of the transmission. Unexpected upon the specified information exchange capacity of the execution, certain advances are needed to ensure the leading execution and proficiency. Instead of piling up unused and unnecessary data on the cloud, we can run time sensitive errands on patient's collected data. In addition, the cloud may be used to transfer the complete data to the server in an unexpected case such as brief absence of availability or vitality of obstacles to the mobile phone.

Cloud Preparing has three unmistakable segments: stockpiling, examination, and perception. The framework is intended for long haul stockpiling of patient's biomedical data also helping wellbeing experts with indicative data. Cloud based therapeutic information stockpiling and the forthright difficulties have been widely tended to in the writing. Examination that utilization the sensor information alongside e-Wellbeing records that are getting to be predominant can help with judgments and guesses for various wellbeing conditions and infections. Moreover, Representation is a key prerequisite for several such structures since it is unrealistic to request that doctors pore over the capacious information or investigations from different wearable of sensors. Representation techniques that are make information examinations available to them for in a promptly edible organization are basic if the wearable different sensors are to affect clinical carry out practice.

Since sensor information is gathered, put away and prepared consistently, clients can get an all-encompassing photo of the circumstance and convey activities appropriately. Normal applications in view of wellbeing information gathered by sensors can be remote wellbeing observing, brisk response to crisis circumstances or wellbeing information social affair and measurements. Since sensor information is gathered, put away and prepared persistently, clients can get a comprehensive photo of the circumstance and convey activities appropriately. Average applications in view of wellbeing information gathered by sensors can be remote wellbeing checking, snappy response to crisis circumstances or wellbeing information get-together and insights. 


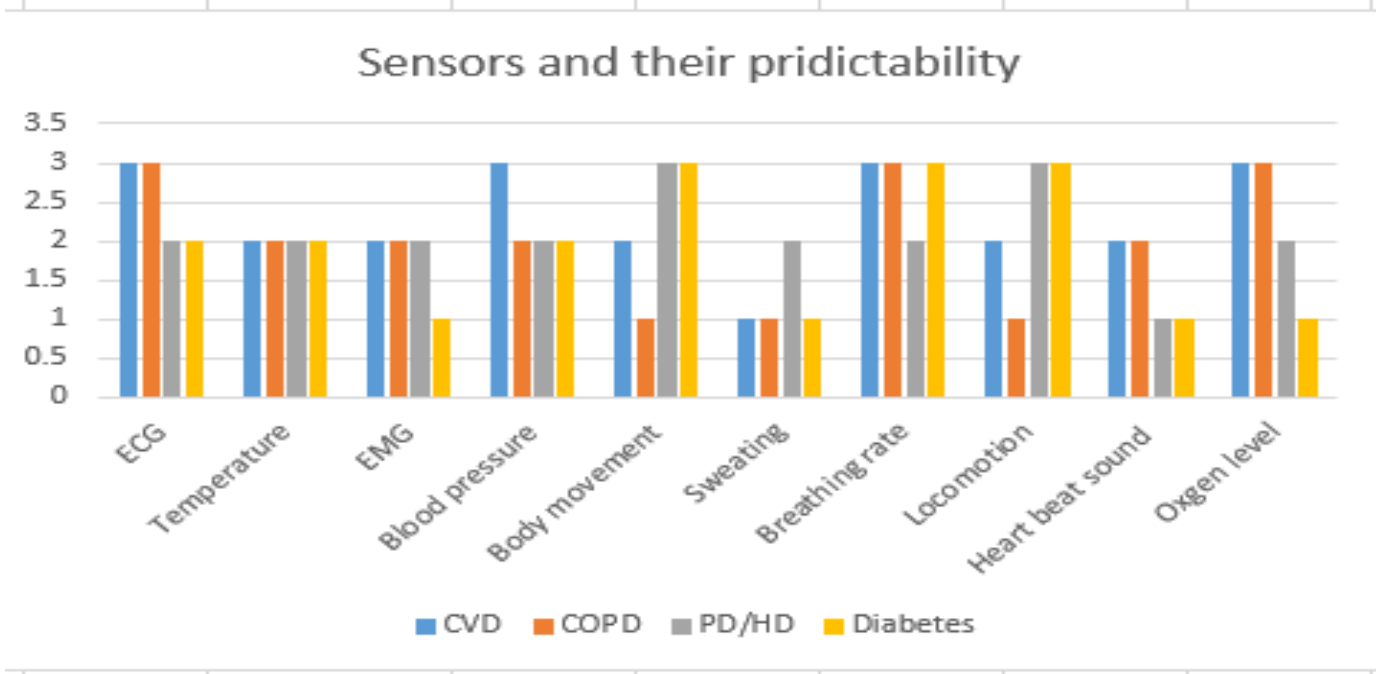

Figure 2 Ailment classification

Fig 2 is a rundown of accessible (best) and future (base) sensors and their relevance to distinguishing wellbeing conditions identified with three basic ailment classifications: cardiovascular ailments (cvd), interminable obstructive aspiratory sickness and parkinson's/huntington's maladies. demonstrates standard appropriateness, shows some materialness, shows undecided appropriateness.

\subsection{Data Acquisition and Sensing}

The sensors should be light, compact and should not impede the creation or flexibility of a patient health monitoring. Similarly, since they must operate on small batteries in the wearable kit, vitality must be effective. In order to avoid data from being misplaced when a rechargeable or reusable battery is being replaced or energized, we need to be allowed extended terms of ceaseless movement without requiring charging. Various sensors can be set as in fig 3 within wearable devices to make contact with skin for therapeutic applications to as to get a estimation of physiological parameters with more precision.
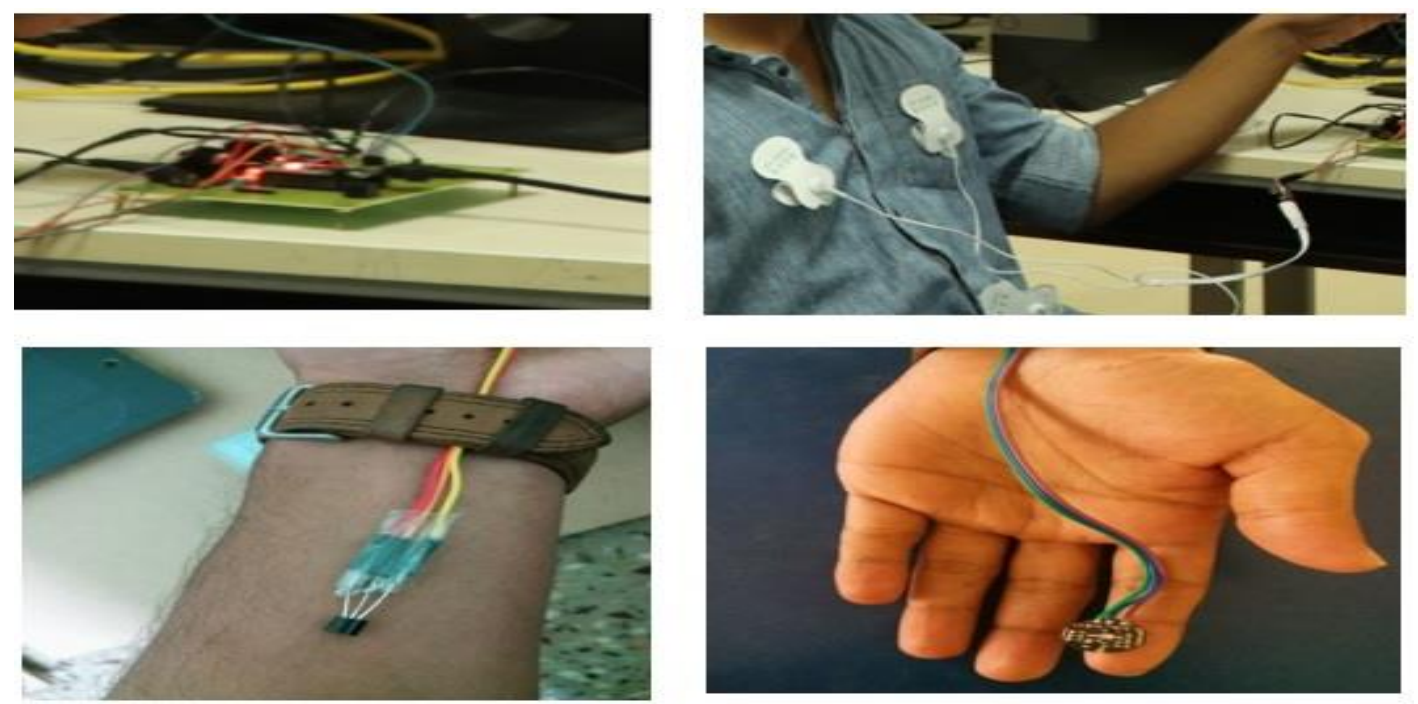

Figure 3 Data collection through wearable devices 
ZigBee over IEEE 802.15.4 is generally utilized as a part of small rate WPANs (LRWPANs) to help correspondence among small power gadgets so as to work in individual working space (POS) of roughly $10 \mathrm{~m}$. ZigBee furnishes dependable work organizing with expanded battery life.

\subsection{Processing of Data Storage by Cloud}

Data gathered by the concentrator ought to be traded in the direction of the cloud as in fig 4 for long pull stockpiling. Releasing the stockpiled data onto the cloud helps in making the system open and flexible when asked for some details by the patients or clinical establishments with current information to educate the doctor's present forecasts/analyze. The procedure would then be able to iterate.

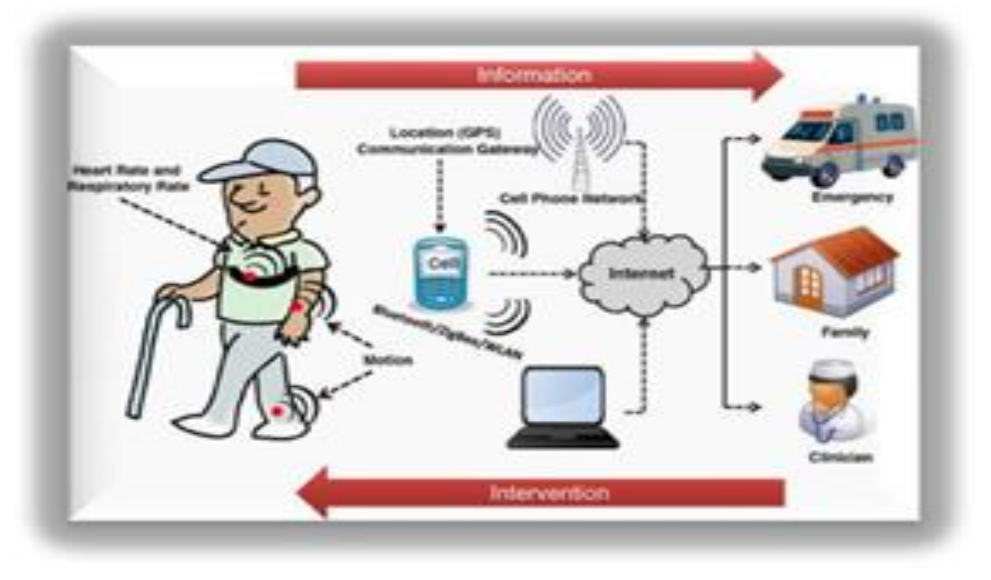

Figure 4 Connectivity

The benefit of making the linkage in the company of the clinical proceedings is that progressing clinical carry out can give preparing information to the machine learning with almost no extra weight on the doctors.

\subsection{Data Analytics}

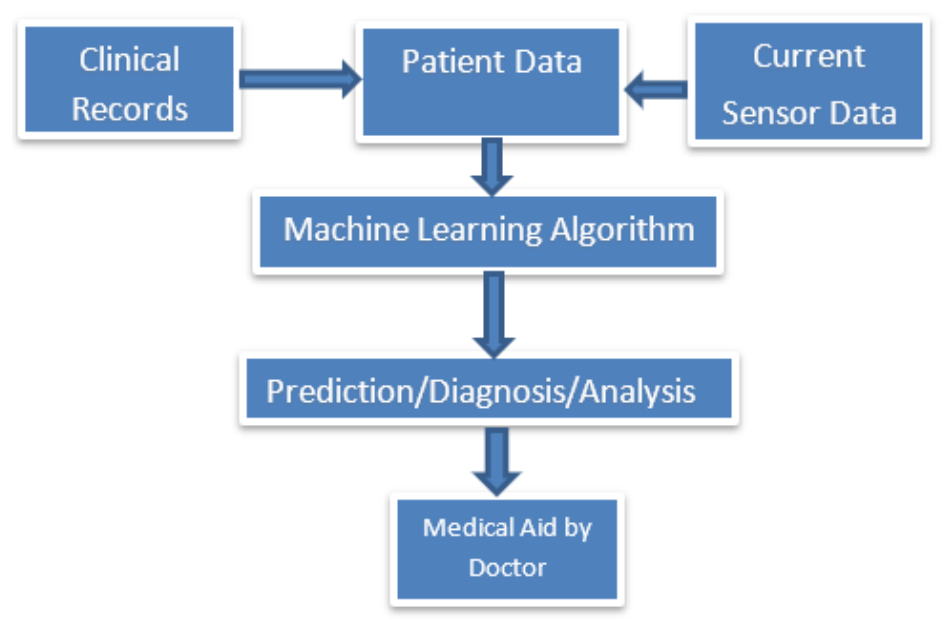

Figure 5 Workflow Diagram.

The doctor will have access to stockpiled data of patients stored in the cloud with a welldefined clinically accepted metrics. The complete workflow is as shown in fig 5 . The doctor 
will be able to analyze the physiological circumstances of patients based on current sensor data which is traded to the cloud for taking immediate and effective action that in turn intensify the wellbeing of patients.

\section{VISUALIZATION}

Data visualization is one of the major advancements provides analysis at various levels of details which helps to make decisions quickly. Shading separation and shading class have been appeared to be viable in permitting fast identification and understanding of contrasts in all intents and purposes. The model will continuously collect millions of records data pertaining to the patient. A sample of data collected is as shown in the table 1 . The sort of graphical form is the best subject to the kind of information visualization drill down through the layers of details as shown in fig 6.

Table 1 Sample data

\begin{tabular}{|c|c|c|c|c|c|c|c|c|c|c|c|}
\hline SI. No. & $\begin{array}{l}\text { Sensors ( threshold values) - } \\
\text { Readings }\end{array}$ & & & & & & & & & & \\
\hline 1 & $\begin{array}{l}\text { Blood Pressure Sensor-Arterial } \\
\text { system ( } 30 \text { to } 300 \mathrm{mmh} \text { ) }\end{array}$ & 60 & 64 & 57 & 69 & 85 & 92 & 71 & 80 & 54 & 72 \\
\hline 2 & Veinous system (5-15) & 6 & 5 & 6 & 6 & 6 & 6 & 7 & 9 & 6 & 7 \\
\hline 3 & Pulmonary system (6-25mmhg) & 12 & 11 & 12 & 11 & 11 & 11 & 9 & 13 & 11 & 12 \\
\hline 4 & $\begin{array}{l}\text { Oxygen level sensor }(80-90 \mathrm{mmhg}) \\
\text { resting adult }>65 \text { years }\end{array}$ & 81 & 84 & 84 & 88 & 80 & 88 & 88 & 88 & 87 & 88 \\
\hline 5 & $\begin{array}{c}\text { ECG Sensor (.08 -0.1 p wave) (0.1- } \\
.20 \text { pr interval)(Qrs } 0.06-0.1) \\
\end{array}$ & 0.09 & 0.1 & 0.1 & 0.15 & 0.12 & 0.18 & 0.11 & 0.09 & 0.1 & 0.1 \\
\hline 6 & Pulse Sensor(65-140) & 90 & 89 & 100 & 91 & 92 & 93 & 93 & 95 & 88 & 102 \\
\hline 7 & Body temp sensor Celsius & 34 & 34.2 & 34.8 & 35.3 & 35.9 & 34.1 & 34.5 & 34.8 & 33.9 & 35.4 \\
\hline 8 & EEG $(0.1-100 \mathrm{hz})$ & 22 & 35 & 33 & 36 & 29 & 28 & 37 & 41 & 42 & 48 \\
\hline 9 & EMG $(.5-2 \mathrm{mv})$ & 0.6 & 0.9 & 0.9 & 0.8 & 0.8 & 0.7 & 0.7 & 0.8 & 0.9 & 0.9 \\
\hline
\end{tabular}

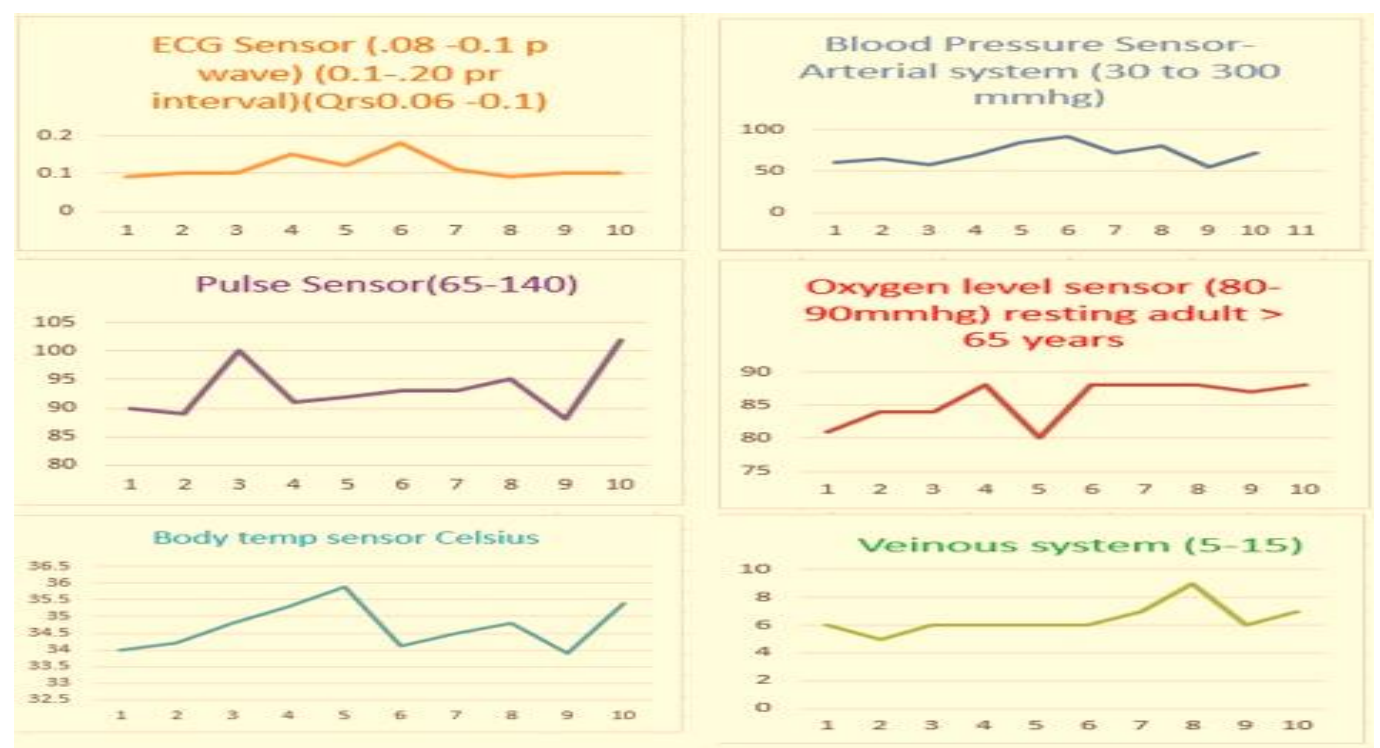

Figure 6 Graph of visualization of Patients health data analysis

\section{CONCLUSION}

Beginning with present status and anticipated prospect bearings for coordination of isolated wellbeing checking advances into the pharmaceutical clinical routine. Wearable sensors, particularly IoT sensors, offer attractive options for recognition and recording in domestic and 
working environments of information or data, over a long period of time than displayed in regenerative offices, as well as for requests for center visits. It promotes a superior way of life by providing customized and streamlined administrations. It should be used after identification, analysis and interpretation before structures can be intended for a specific mix of clinical practice. Factors of a human body, both internal and external can be checked and assessed. These factors along with genomic inputs help predict the wellbeing patterns and hypersensitivities of the individual. This way, suggestions can be given as to what kind of exercise and diet must be followed.

\section{REFERENCES}

[1] A. Pantelopoulos and N. Bourbakis, (2010) "A survey on wearable sensor-based systems for health monitoring and prognosis," IEEE Trans. Sys., Man, and Cybernetics, Part C: Applic. and Reviews, vol. 40, no. 1, pp. 1-12.

[2] Aleksandar Kotevski1, Natasa Koceska2 and Saso Koceski, (2016)" E-health monitoring system" International conferece on applied internet and information technologies.

[3] J. Jin, J. Gubbi, S. Marusic, and M. Palaniswami, (2014) "An information framework for creating a smart city through internet of things," IEEE Internet of Things Journal, vol. 1, no. 2, pp. 112-121.

[4] M. Fengou, G. Mantas, D. Lymberopoulos, N. Komninos, S. Fengos, and N. Lazarou, (2013) "A new framework architecture for next generation ehealth services," Biomedical and Health Informatics, IEEE Journal of, vol. 17, no. 1, pp. 9-18.

[5] D. Lake, R. Milito, M. Morrow, and R. Vargheese, (2014) "Internet of things: Architectural framework for ehealth security," Journal of ICT Standardization, River Publishing, vol. 1.

[6] A. Chehri, H. Mouftah, and G. Jeon, (2010) "A smart network architecture for e-health applications," in Intelligent Interactive Multimedia Systems and Services. Springer Berlin Heidelberg, pp. 157-166.

[7] Sneha N. Malokar, Samadhan D. Mali (2017) "A IoT Based Health Care Monitoring System" International Journal of Advanced Research in Electrical, Electronics and Instrumentation Engineering Vol. 6, Issue 6.

[8] M. Sathya, S. Madhan, K. Jayanthi (2018) "Internet of things (IoT) based health monitoring system and challenges" International Journal of Engineering and Technology, 7 (1) $175-178$ 